\title{
Revista Colombiana de

\section{Capítulo 7. Utilidad del mapeo tridimensional en la ablación de la fibrilación auricular persistente}

\section{Chapter 7. Usefulness of three-dimensional mapping in persistent atrial fibrillation}

\author{
William Uribe*, Julián M. Aristizábal, Juan C. Díaz, Jorge E. Velásquez, Jorge E. Marín \\ y Mauricio Duque
}

Servicio CES Cardiología, Medellín, Colombia

Recibido el 16 de noviembre de 2015; aceptado el 18 de enero de 2016

\section{Introducción}

La fibrilación auricular es la arritmia más común en la práctica clínica y es responsable de un número importante de hospitalizaciones, morbilidad, mortalidad y costos en atención médica ${ }^{1}$. Desde finales de los setenta, se sabe que el paciente con fibrilación auricular tiene un riesgo mayor de eventos embólicos (aproximadamente cinco veces el de la población general, 17 veces en pacientes con estenosis mitral). Es así como a principios de la década de los noventa aparecieron los primeros estudios de anticoagulación con warfarina para la prevención de eventos embólicos en fibrilación auricular ${ }^{2}$. En la última década, la aparición de nuevos anticoagulantes orales ha brindado alternativas para la anticoagulación de estos pacientes. No obstante, si bien la anticoagulación logra disminuir de manera significativa la tasa de eventos embólicos y la morbi-mortalidad asociada, no tiene ningún impacto en la evolución de la enfermedad. Es decir, se previenen las complicaciones mas no se da tratamiento a la patología.

Se han usado diversos fármacos para lograr mantener el ritmo sinusal, con una baja tasa de éxito en la mayoría de los casos y con un número elevado de efectos adversos asociados, lo que ha hecho que la estrategia de control de ritmo no muestre ningún beneficio sobre la estrategia de control de la frecuencia ${ }^{3-6}$. Desde entonces, la noción de que la estrategia de control del ritmo es igual a la de control de la respuesta ventricular ha obtenido una difusión marcada ${ }^{7}$ e, infortunadamente en muchos casos, ha impedido que pacientes que pudieran retornar fácilmente al ritmo sinusal no reciban el tratamiento apropiado. Modelos animales han demostrado cómo la fibrilación auricular induce cambios eléctricos en aurículas que favorecen el sostenimiento de la arritmia, lo cual permitió acuñar la frase "la fibrilación auricular genera fibrilación auricular"8. Es decir, la estrategia de control de la frecuencia, si bien es segura y deseable en un grupo de pacientes, no puede ser aplicada de manera generalizada ya que condena a otros que podrían retornar a ritmo sinusal a permanecer en fibrilación auricular.

En 1998 se demostró la importancia de las venas pulmonares en la génesis de la fibrilación auricular ${ }^{9}$ y a partir de ese momento el aislamiento de las venas pulmonares se ha posicionado como una terapia efectiva y segura para el manejo de la arritmia. A medida que la arritmia perdura, los cambios estructurales y eléctricos que sufre la aurícula izquierda hacen que las venas pulmonares pierdan su preponderancia en la génesis de la arritmia (como sucede en la fibrilación

*Autor para correspondencia.

Correo electrónico: william.uribe.md@gmail.com (W. Uribe). 
auricular paroxística) y comiencen a aparecer distintos disparadores como la pared posterior de la aurícula y las venas torácicas (vena cava superior, seno coronario, vena de Marshall $)^{10,11}$. Esto es particularmente importante en pacientes con fibrilación auricular persistente (es decir, la que tiene una duración de más de 7 días o que requiere de una intervención médica para su terminación), lo cual tiene un impacto significativo en la tasa de éxito del aislamiento de las venas pulmonares como estrategia única del manejo.

A pesar de ello, en el caso de la fibrilación auricular persistente la ablación con catéter es una estrategia razonable para el manejo del paciente sintomático con una arritmia refractaria al tratamiento antiarrítmico, con una recomendación lla y nivel de evidencia $B$ según las últimas recomendaciones del consenso de expertos ${ }^{12}$. La ablación con radiofrecuencia constituye la alternativa más utilizada (en comparación con otras modalidades, principalmente la crioablación) sustentada en gran número de pacientes reportados en la literatura. En estos casos, el aislamiento de la pared posterior de la aurícula izquierda y la eliminación de focos automáticos disparadores de la arritmia parecen tener un lugar destacado en el mantenimiento del ritmo sinusal, con algunos estudios que muestran además beneficios de la eliminación de potenciales fraccionados ${ }^{13-16}$. Otras estrategias estudiadas (con menor número de pacientes y menor evidencia que avale su utilidad) incluyen el aislamiento de la orejuela izquierda y la ablación de rotores ${ }^{17,18}$.

Si bien el aislamiento de las venas pulmonares como estrategia única para el manejo de la fibrilación auricular puede hacerse bajo visualización fluoroscópica y ecocardiográfica, las estrategias mencionadas para el manejo de la fibrilación auricular persistente requieren otro tipo de mapeo, que permita la reconstrucción de la cavidad auricular y determinen de manera precisa las características y ubicación del blanco terapéutico (sea este la pared posterior, los focos automáticos disparadores o los potenciales fraccionados). Es aquí donde los sistemas de mapeo tridimensional han aportado grandes avances en el área de la electrofisiología, ya que favorecen un mejor entendimiento de los mecanismos que permiten la progresión de la fibrilación auricular paroxística hacia la fibrilación auricular persistente, así como la perpetuación de la arritmia a través de los fenómenos de remodelación. Además, la integración de los mismos con técnicas de imágenes como la tomografía computarizada (TC), la resonancia magnética (RM), la ecocardiografía intracardiaca o la angiografía rotacional aumenta la precisión con la cual se delimitan las estructuras anatómicas, facilitando la manipulación de los catéteres dentro de ellas sin necesidad de visualización fluoroscópica. Vale la pena resaltar que el uso de estas fusiones disminuye el tiempo de fluoroscopia durante la fase de reconstrucción de la cavidad auricular izquierda pero no afecta el resultado final ${ }^{19}$. Es decir, la reconstrucción tridimensional se puede llevar a cabo sin la fusión a expensas de un aumento en el tiempo de fluoroscopia.

Estas características han hecho que los sistemas de navegación hayan obtenido una gran aceptación mundial en muy poco tiempo, de tal manera que alrededor del $90 \%$ del grupo de expertos utiliza rutinariamente sistemas de mapeo electroanatómico para la ablación de la fibrilación auricular ${ }^{12}$. Es posible que para la ablación de la fibrilación auricular persistente y la fibrilación auricular largamente persistente, este porcentaje sea aún más elevado.

\section{Utilidad del mapeo tridimensional}

\section{Características de los diferentes sistemas de navegación tridimensional y su impacto en la ablación de la fibrilación auricular persistente}

Actualmente en Colombia se cuenta con los sistemas EnSite ${ }^{\circledR}$ y CARTO ${ }^{\circledR}$, los cuales utilizan distintas tecnologías para determinar la posición del catéter dentro de la aurícula.

El sistema EnSite (St. Jude Medical, St. Paul, Minnesota) utiliza una pequeña corriente $(5,6 \mathrm{KHz})$ entre parches ortogonales en varias ubicaciones en el tórax del paciente y luego algoritmos de triangulación determinan la posición del catéter dentro del corazón (mas no la orientación exacta de la punta ni tampoco los efectos del torque y la rotación del catéter) y la actividad eléctrica local registrada por los catéteres dentro de las cavidades. El sistema puede determinar la posición de varios catéteres de manera simultánea, incluso cuando son de otro fabricante, lo cual facilita el uso de distintas herramientas durante el proceso de ablación, siendo esta una de sus principales ventajas. Es compatible para la integración con TC, RM, angiografía rotacional y ecocardiografía intracardiaca (figs. 1-3).

En pacientes con fibrilación auricular persistente o recurrencias, según la aproximación utilizada por el electrofisiólogo, permite mapear la posible reconexión de las venas pulmonares; realizar un mapa de voltaje para determinar la presencia de tejido cicatricial y posibles brechas que hayan generado las recurrencias; realizar, mediante un software especial presente en el equipo, el mapeo de los CAFE (electrogramas atriales complejos fraccionados, por su sigla en inglés) para efectuar ablación dirigida hacia su localización, y mapear las macrorreentradas o arritmias focales que puedan estar presentes en la recurrencia.

El sistema es muy sensible a los cambios en la impedancia transtorácica, por lo cual se debe tener en cuenta el hábito

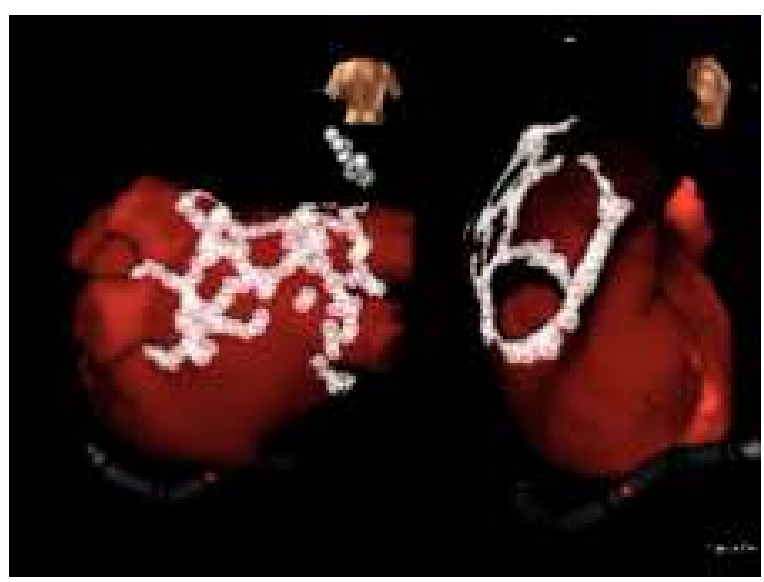

Figura 1 Reconstrucción 3-D de la aurícula izquierda e integración con angioTAC multicortes mediante sistema EnSite NavX. Los puntos blancos muestran el territorio sometido a la ablación en un paciente con fibrilación auricular persistente. Se observa ablación de los antros de las venas pulmonares, la pared posterior, el techo y la vena cava superior. Imagen del laboratorio de Electrofisiología CES Cardiología. 


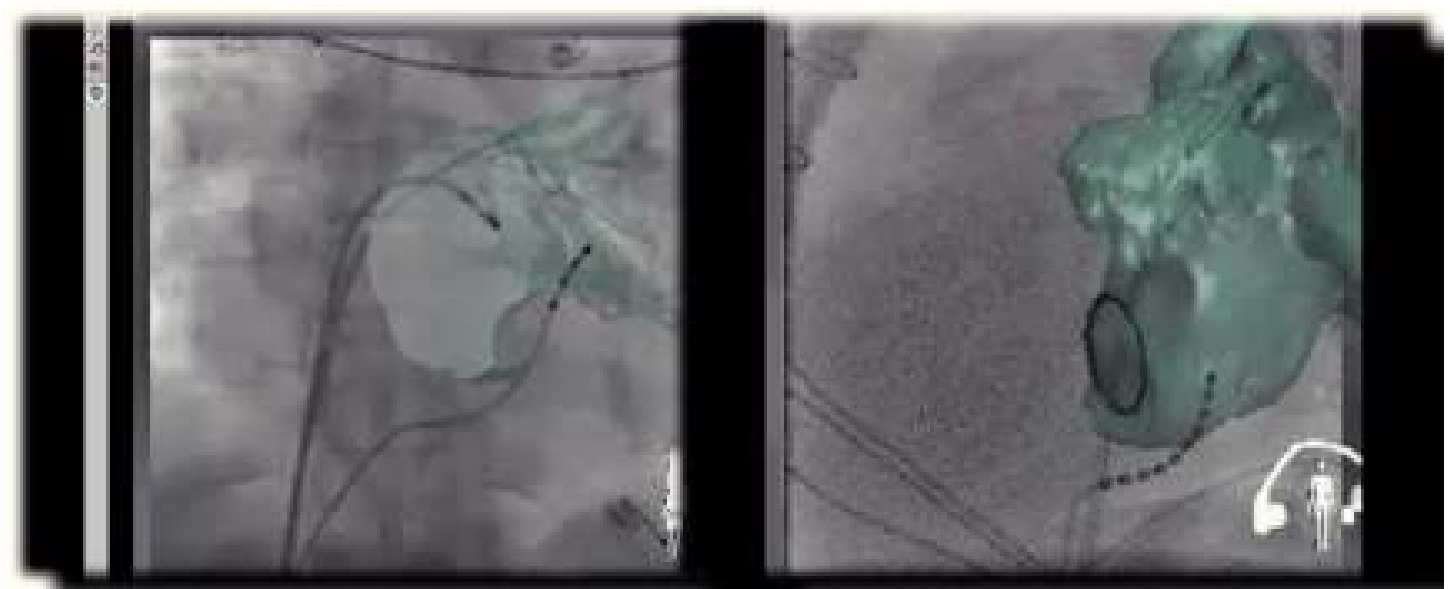

Figura 2 Reconstrucción tridimensional en la que se utiliza angiografía rotacional y fluoroscopia. Se fusiona la angioTAC con la imagen fluoroscópica a través de un software (EP navigator ${ }^{\circledR}$ de Philips). Permite la visualización en tiempo real de los catéteres dentro de la cámara estudiada, en este caso la aurícula izquierda.

corporal del paciente (hay restricciones de peso y tamaño). Los cambios en los fluidos corporales con la aparición de edema también cambian la impedancia transtorácica durante el procedimiento, lo cual, en teoría, podría afectar la precisión del sistema durante procedimientos prolongados (como podría ser el caso durante una ablación de una fibrilación auricular persistente). Nuevamente, esta afirmación se basa en conceptos teóricos y su impacto no ha sido determinado en la práctica, de modo que a la luz de la evidencia actual no debe tenerse en cuenta para la toma de decisión en cuanto a qué sistema escoger durante la ablación.

Por su parte, el sistema CARTO ${ }^{\circledR}$ (Biosense Webster, Diamond Bar, California, Estados Unidos) utiliza un campo magnético de bajo poder emitido por tres bobinas ubicadas bajo el tórax del paciente, el cual en conjunto con el sensor magnético en la punta del catéter permite su ubicación exacta

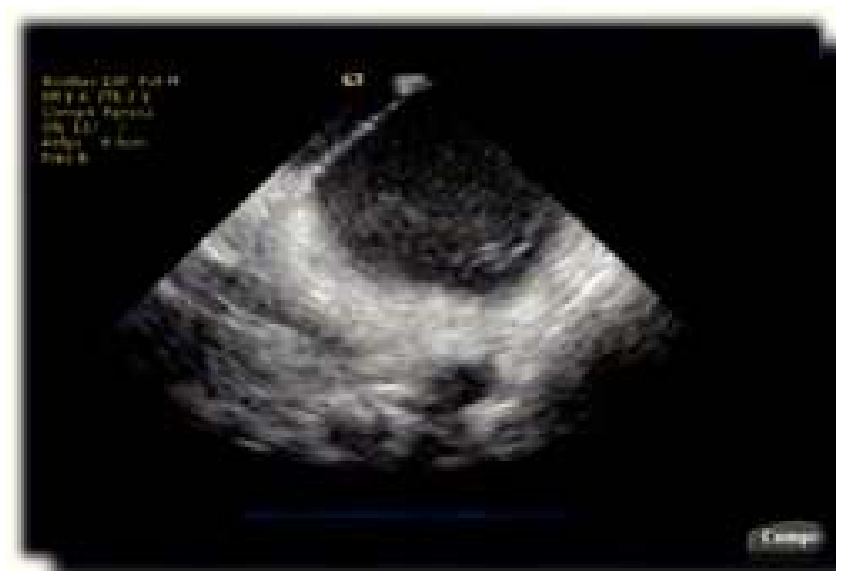

Figura 3 Ecocardiografía intracardiaca. Imagen de la aurícula izquierda en tiempo real durante un procedimiento de ablación de fibrilación atrial persistente en donde se logra visualizar el septum interauricular y la cavidad auricular izquierda con un catéter en la proximidad del antro de las venas pulmonares izquierdas. dentro del corazón, incluyendo la orientación de la punta del catéter y los efectos de rotación del mismo, característica que permite establecer la posición del catéter con una resolución menor a $1 \mathrm{~mm}$. Los electrogramas registrados son acoplados con la localización del paciente para crear la anatomía tridimensional. Permite realizar mapeo anatómico, de voltaje, activación y propagación. Es compatible para la integración con TC, RM, angiografía rotacional y ecocardiografía intracardiaca (figs. 4 y 5). Mediante el sistema CARTOSOUND $^{\circledR}$, la reconstrucción tridimensional de la aurícula puede realizarse con las imágenes obtenidas por la sonda de ecocardiografía intracardiaca. Los catéteres utilizados con el sistema CARTO tienen en su punta un sensor compuesto por coils miniaturizados, por lo que no se ve afectado por estos cambios de impedancia torácica. Esta capacidad de ser detectado por el campo magnético es propia de los catéteres para sistema CARTO que poseen un magneto en su punta, por lo que no es posible determinar la posición de los catéteres convencionales, limitando el uso de estos con dicho sistema de navegación.

Independiente del tipo de sistema que se use, ambos se asocian a una disminución del tiempo de fluoroscopia. Se ha sugerido que el sistema CARTO admite un menor tiempo de fluoroscopia respecto al sistema EnSite, probablemente en relación con la información que brinda sobre el grado de rotación y la orientación de la curva del catéter; estos resultados deben ser corroborados en estudios de mayor tamaño con el fin de dar un dictamen final sobre la superioridad de un sistema sobre el otro $^{20}$. De manera simultánea, ambos sistemas permiten la creación de registros con información anatómica y eléctrica, produciendo mapas de activación, voltaje y propagación.

Los dos sistemas han desarrollado aplicaciones complementarias de tecnología de contacto (Contact Force para el sistema CARTO y Tacticath para el sistema EnSite) $)^{21,22}$ que permiten establecer en tiempo real la fuerza de contacto entre la punta del catéter y la superficie cardiaca, característica determinante en la eficacia y particularmente en la seguridad de los procedimientos, ya que deja adaptar los parámetros físicos de la aplicación de radiofrecuencia al grama- 

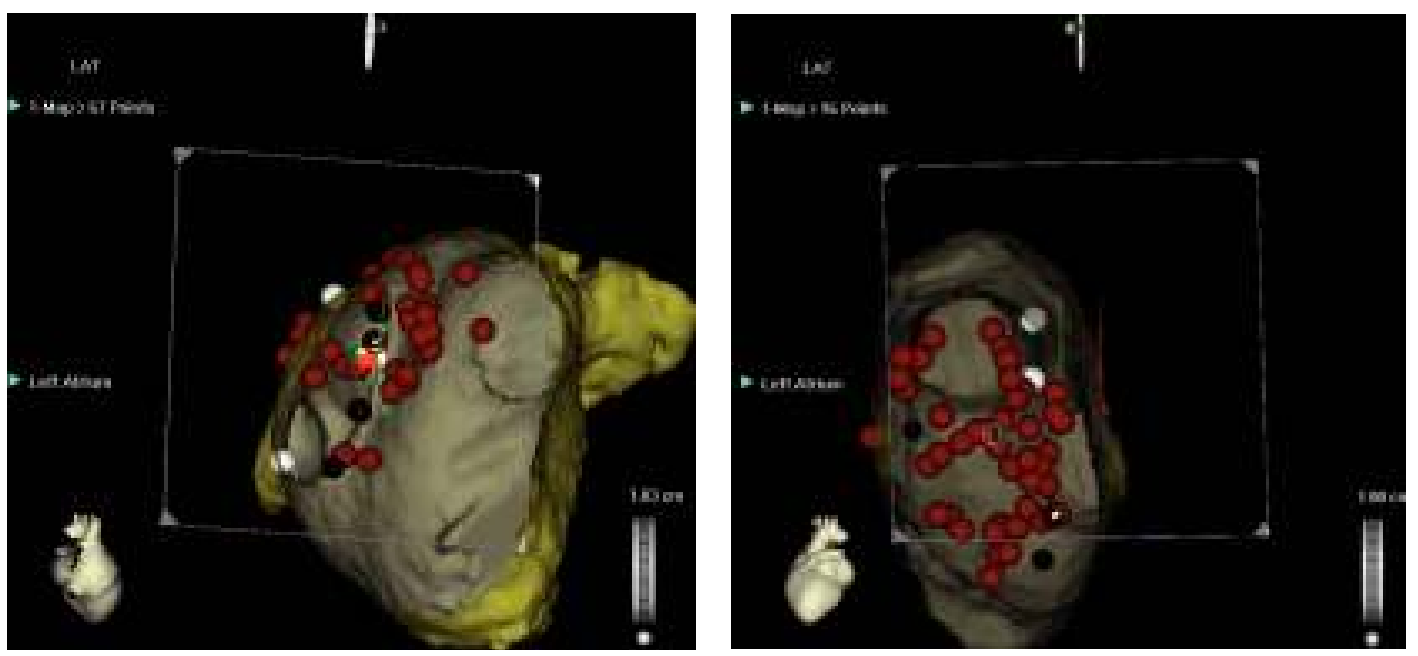

Figura 4 Izquierda: reconstrucción tridimensional de la aurícula izquierda después de integración con angioTAC multicortes en el sistema CARTO que permite visualización endoscópica virtual del antro común de las venas pulmonares izquierdas. Los puntos rojos señalan los sitios de aplicación de radiofrecuencia. Se observan las venas pulmonares izquierdas, superior e inferior y la orejuela. Derecha: Visualización endoscópica virtual del antro de las venas pulmonares derechas, superior e inferior. Imagen del laboratorio de Electrofisiología CES Cardiología.

je de la fuerza de contacto reportada por el sistema. Está claramente definido que aplicaciones con menos de 10 gramos de presión son ineficaces y determinan recurrencias, y que aquellas con más de 40 gramos de presión incrementan dramáticamente la tasa de perforaciones y taponamiento cardiaco. Los estudios han demostrado el impacto de esta tecnología en la eficacia, la seguridad y los tiempos del procedimiento, así como en la reducción en la exposición a radiación ionizante.

\section{Beneficios del uso de sistemas de navegación tridimensional en la ablación de fibrilación auricular persistente}

Actualmente, los sistemas de mapeo tridimensional brindan varias ventajas sobre la fluoroscopia en la ablación de la fibrilación auricular persistente:

- La utilización de los sistemas tridimensionales reducen el tiempo de fluoroscopia ${ }^{23-26}$. A pesar de no existir estudios sobre el riesgo de cáncer en pacientes con ablación de fibrilación auricular, se sabe que la fluoroscopia aumenta este riesgo existiendo una relación lineal entre la dosis medida en milisieverts y el riesgo de desarrollar cáncer, particularmente de mama ${ }^{27}$. Existe una alta probabilidad de que se realicen varios estudios imagenológicos utilizando energía ionizante (tomografías, radiografías, fluoroscopias) a un mismo paciente, por lo cual es deseable la disminución del tiempo de exposición durante procedimientos de ablación ya que la dosis (y por ende el riesgo) es acumulativa.

- Permiten la reconstrucción tridimensional de la anatomía de la aurícula izquierda creando de manera simultánea mapas anatómicos y de voltaje, activación o propagación.

- Disminuyen el riesgo de estenosis de venas pulmonares mediante la delimitación adecuada del ostium y antro de las mismas.
- Facilitan la ablación en pacientes con variaciones anatómicas como venas adicionales, anguladas o en sitios que requieren delimitación muy precisa, como las carinas ${ }^{28}$.

- Disminuyen la presencia de brechas durante el aislamiento de las venas pulmonares. El registro del territorio sometido a la ablación permite cerrar la brecha visual que se ha relacionado con reconexión de las venas pulmonares ${ }^{29}$.

- Brindan la posibilidad de guiar la modificación del sustrato atrial mediante el mapeo de voltaje, electrogramas fraccionados y mapeo de CAFE (complex atrial fractionated electrograms). Permiten además la ablación de estructuras cuya ubicación fluoroscópica es difícil, como por ejemplo la vena de Marshall o los focos automáticos disparadores.

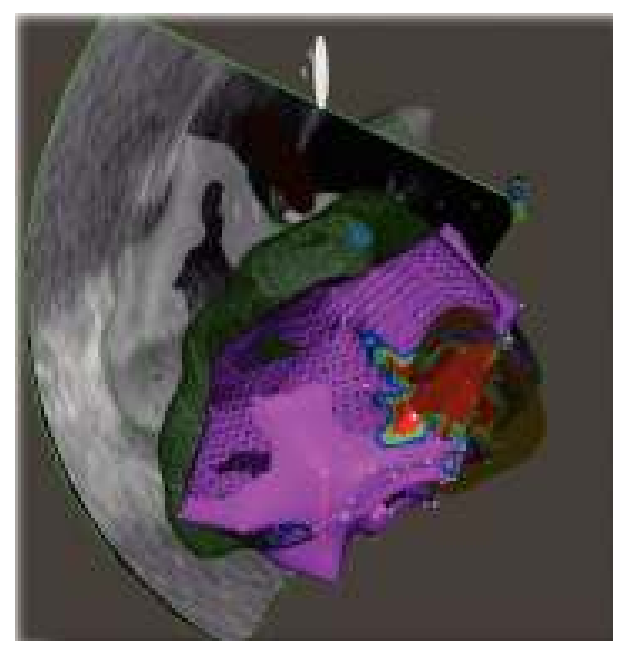

Figura 5 Reconstrucción tridimensional con la tecnología CARTOSOUND. En este tipo de reconstrucción se utiliza un catéter que en su punta tiene la combinación de un transductor miniaturizado para ecocardiografía intracardiaca y el sistema magnético de CARTO. 
- Facilitan el mapeo de las macrorreentradas y taquicardias focales durante recurrencias. El mapeo de activación permite definir con precisión el origen y la diseminación de las arritmias encontradas en las recurrencias, delimitar los circuitos de las reentradas y verificar la reconexión de las venas pulmonares.

Teniendo en cuenta los cambios eléctricos y estructurales que ocurren en la aurícula izquierda a medida que se permanece más tiempo en fibrilación auricular, no es de extrañar que los resultados de la ablación de fibrilación auricular persistente no sean tan buenos como los resultados de la ablación en fibrilación auricular paroxística. En los Estados Unidos, la ablación para al manejo de fibrilación auricular persistente no tiene aprobación de la Administración de Drogas y Alimentos (FDA, su sigla en inglés) (distinta a su aplicación con fines investigativos) y de manera reciente ha estado bajo escrutinio en países como Bélgica o el Reino Unido. A pesar de esto, en el mundo cerca del $86 \%$ de los centros realizan ablación en pacientes con fibrilación auricular persistente, y un $47 \%$ utiliza la ablación para el manejo de pacientes con fibrilación auricular persistente de larga duración ${ }^{30}$.

La tasa de éxito es claramente menor en pacientes con fibrilación auricular persistente: aproximadamente 63\% están libres de antiarrítmicos y de fibrilación auricular a los 18 meses de seguimiento en un registro mundial ${ }^{30}$. Estos datos son confirmados por un metaanálisis reciente, en el que se encontró una tasa de éxito del $68 \%$, siendo mayor cuando se logra aislamiento eléctrico ( $78 \%$ de efectividad) ${ }^{31}$. De manera interesante, en este metaanálisis se encontró que la creación de líneas adicionales (entre la válvula mitral y la vena pulmonar inferior izquierda o línea de techo) disminuía de manera significativa la tasa de recurrencias, con una tasa de efectividad del $78 \%$. No obstante, hubo heterogeneidad moderada de los estudios y diferencias significativas en el tipo de línea creada, por lo cual estos resultados deben ser tomados con precaución ${ }^{31}$.

Sin embargo, la creación de líneas adicionales o la ablación de café no ha demostrado en un estudio aleatorizado reciente ningún beneficio en cuanto al mantenimiento del ritmo sinusal a 18 meses, asociándose en cambio a un mayor tiempo de fluoroscopia y duración total del procedimiento ${ }^{32}$, por lo que hay gran duda en cuanto a la utilidad de estos procedimientos.

\section{Conclusiones}

Los sistemas de navegación tridimensional desempeñan un papel determinante en la ablación de la fibrilación auricular persistente, pues logran disminuir los tiempos de fluoroscopia y del procedimiento. Adicionalmente, permiten establecer blancos de ablación (venas torácicas, CAFE, focos automáticos disparadores, pared posterior de la aurícula izquierda) que no sería posible identificar mediante fluoroscopia. En el caso particular de la fibrilación auricular persistente, la ablación de estas estructuras puede tener un impacto significativo en el desenlace a mediano y largo plazo, con una disminución en las tasas de recurrencias, de ahí que los sistemas de ablación deban usarse siempre durante la ablación de esta arritmia idealmente asociados al uso de ecografía intracardiaca y tecnología de fuerza de contacto.

\section{Recomendaciones}

\section{Clase I}

- El abordaje integral del sustrato arrítmico en fibrilación auricular persistente requiere del uso de los sistemas de mapeo tridimensional idealmente asociado al empleo de ecografía intracardiaca.

- Se recomienda el uso de tecnologías de mapeo tridimensional durante la ablación de fibrilación auricular persistente con menor irradiación para el paciente y mejor aproximación anatómica de la aurícula izquierda, definición de líneas de ablación y detección de CAFE (nivel de evidencia C).

- Se recomienda el uso de tecnologías de mapeo tridimensional durante la aplicación de técnicas de ablación distintas al aislamiento de venas pulmonares, como la ablación de CAFE, la ablación de otras venas torácicas y la creación de líneas adicionales (nivel de evidencia $\mathrm{C}$ ).

\section{Bibliografía}

1. Mozaffarian D, Benjamin EJ, Go AS, et al. Heart disease and stroke statistics-2015 update: a report from the american heart association. Circulation. 2015;131:e29-322.

2. Wolf PA, Dawber TR, Thomas HE Jr, Kannel WB. Epidemiologic assessment of chronic atrial fibrillation and risk of stroke: the Framingham study. Neurology 1978;28:973-7.

3. Wyse DG, Waldo AL, DiMarco JP, et al. A comparison of rate control and rhythm control in patients with atrial fibrillation. $\mathrm{N}$ Eng J Med. 2002;347:1825-33.

4. Van Gelder IC, Hagens VE, Bosker HA, et al. A comparison of rate control and rhythm control in patients with recurrent persistent atrial fibrillation. N Eng J Med. 2002;347:1834-40.

5. Hohnloser SH, Kuck KH, Lilienthal J. Rhythm or rate control in atrial fibrillation--Pharmacological Intervention in Atrial Fibrillation (PIAF): a randomised trial. Lancet. 2000;356:1789-94.

6. Carlsson J, Miketic S, Windeler J, et al. Randomized trial of rate-control versus rhythm-control in persistent atrial fibrillation: the Strategies of Treatment of Atrial Fibrillation (STAF) study. J Am Coll Cardiol. 2003;41:1690-6.

7. Falk RH. Is rate control or rhythm control preferable in patients with atrial fibrillation? Rate control is preferable to rhythm control in the majority of patients with atrial fibrillation. Circulation. 2005;111:3141-50; discussion 3157.

8. Wijffels MC, Kirchhof CJ, Dorland R, Allessie MA. Atrial fibrillation begets atrial fibrillation. A study in awake chronically instrumented goats. Circulation. 1995;92:1954-68.

9. Haissaguerre $M$, Jais $P$, Shah DC, et al. Spontaneous initiation of atrial fibrillation by ectopic beats originating in the pulmonary veins. N Eng J Med. 1998;339:659-66.

10. Hwang C, Wu TJ, Doshi RN, Peter CT, Chen PS. Vein of marshall cannulation for the analysis of electrical activity in patients with focal atrial fibrillation. Circulation. 2000;101:1503-5.

11. Jais $P$, Haissaguerre $M$, Shah $D C$, et al. A focal source of atrial fibrillation treated by discrete radiofrequency ablation. Circulation. 1997;95:572-6.

12. Calkins H, Kuck KH, Cappato R, et al. 2012 HRS/EHRA/ECAS Expert Consensus Statement on Catheter and Surgical Ablation of Atrial Fibrillation: Recommendations for Patient Selection, Procedural Techniques, Patient Management and Follow-up, Definitions, Endpoints, and Research Trial Design: A report of the Heart Rhythm Society (HRS) Task Force on Catheter and Surgical Ablation of Atrial Fibrillation. Developed in partnership with the European Heart Rhythm Association (EHRA), a re- 
gistered branch of the European Society of Cardiology (ESC) and the European Cardiac Arrhythmia Society (ECAS); and in collaboration with the American College of Cardiology (ACC), American Heart Association (AHA), the Asia Pacific Heart Rhythm Society (APHRS), and the Society of Thoracic Surgeons (STS). Endorsed by the governing bodies of the American College of Cardiology Foundation, the American Heart Association, the European Cardiac Arrhythmia Society, the European Heart Rhythm Association, the Society of Thoracic Surgeons, the Asia Pacific Heart Rhythm Society, and the Heart Rhythm Society. Europace. 2012;14:528-606.

13. Kim JS, Shin SY, Na JO, et al. Does isolation of the left atrial posterior wall improve clinical outcomes after radiofrequency catheter ablation for persistent atrial fibrillation?: A prospective randomized clinical trial. Int J Cardiol. 2014;181C:277-83.

14. Oral H, Chugh A, Good E, et al. Randomized comparison of encircling and nonencircling left atrial ablation for chronic atrial fibrillation. Heart rhythm: the official journal of the Heart Rhythm Society. 2005;2:1165-72.

15. Li WJ, Bai YY, Zhang HY, et al. Additional ablation of complex fractionated atrial electrograms after pulmonary vein isolation in patients with atrial fibrillation: a meta-analysis. Circulation Arrhythmia and Electrophysiology 2011;4:143-8.

16. Elayi CS, Verma A, Di Biase L, et al. Ablation for longstanding permanent atrial fibrillation: results from a randomized study comparing three different strategies. Heart rhythm: the official journal of the Heart Rhythm Society. 2008;5:1658-64.

17. Di Biase L, Burkhardt JD, Mohanty P, et al. Left atrial appendage: an underrecognized trigger site of atrial fibrillation. Circulation. 2010;122:109-18.

18. Narayan SM, Krummen DE, Shivkumar K, Clopton P, Rappel WJ, Miller JM. Treatment of atrial fibrillation by the ablation of localized sources: CONFIRM (Conventional Ablation for Atrial Fibrillation With or Without Focal Impulse and Rotor Modulation) trial. J Am Coll Cardiol. 2012;60:628-36.

19. Caponi D, Corleto A, Scaglione M, et al. Ablation of atrial fibrillation: does the addition of three-dimensional magnetic resonance imaging of the left atrium to electroanatomic mapping improve the clinical outcome?: a randomized comparison of Carto-Merge vs. Carto-XP three-dimensional mapping ablation in patients with paroxysmal and persistent atrial fibrillation. Europace. 2010;12:1098-104.

20. Khaykin Y, Oosthuizen R, Zarnett L, Wulffhart ZA, Whaley B, Hill $C$, et al. CARTO-guided vs. NavX-guided pulmonary vein antrum isolation and pulmonary vein antrum isolation performed without 3-D mapping: effect of the 3-D mapping system on procedure duration and fluoroscopy time. J Interv Card Electrophysiol. 2011;30:233-40.
21. Shurrab M, DiBiase L, Briceno D, et al. Impact of contact force technology on atrial fibrillation ablation. J Am Heart Assoc. 2015;4:e002476.

22. Reddik V, Dukkipati S, Neuzil P, et al. Randomized, Controlled Trial of the Safety and Effectiveness of a Contact Force-Sensing Irrigated Catheter for Ablation of Paroxismal Atrial Fibrillation: Result of the TactiCath Contact Force Ablation Catheter Study for Atrial Fibrillation (TOCCASTAR) Study. Circulation. 2015;132:907-15.

23. Scaglione M, Biasco L, Caponi D, et al. Visualization of multiple catheters with electroanatomical mapping reduces $X$-ray exposure during atrial fibrillation ablation. Europace. 2011;13:955-62.

24. Estner HL, Deisenhofer I, Luik A, et al. Electrical isolation of pulmonary veins in patients with atrial fibrillation: reduction of fluoroscopy exposure and procedure duration by the use of a nonfluoroscopic navigation system (NavX). Europace. 2006;8:583-7.

25. Sporton SC, Earley MJ, Nathan AW, Schilling RJ. Electroanatomic versus fluoroscopic mapping for catheter ablation procedures: a prospective randomized study. J Cardiovasc Electrophysiol. 2004;15:310-5.

26. Ferguson JD, Helms A, Mangrum JM, et al. Catheter ablation of atrial fibrillation without fluoroscopy using intracardiac echocardiography and electroanatomic mapping. Circ Arrhythm Electrophysiol. 2009;2:611-9.

27. Howe GR, McLaughlin J. Breast cancer mortality between 1950 and 1987 after exposure to fractionated moderate-dose-rate ionizing radiation in the Canadian fluoroscopy cohort study and a comparison with breast cancer mortality in the atomic bomb survivors study. Radiation Research. 1996;145:694-707.

28. Lo LW, Chen SA. Three-dimensional electroanatomic mapping systems in catheter ablation of atrial fibrillation. Circ J. 2010;74:18-23.

29. Miller MA, d'Avila A, Dukkipati SR, et al. Acute electrical isolation is a necessary but insufficient endpoint for achieving durable PV isolation: the importance of closing the visual gap. Europace. 2012;14:653-60. doi: 10.1093/europace/eus048

30. Cappato R, Calkins H, Chen SA, et al. Updated worldwide survey on the methods, efficacy, and safety of catheter ablation for human atrial fibrillation. Circulation Arrhythmia and Electrophysiology. 2010;3:32-8.

31. Wynn GJ, Das M, Bonnett LJ, Panikker S, Wong T, Gupta D. Efficacy of catheter ablation for persistent atrial fibrillation: a systematic review and meta-analysis of evidence from randomized and nonrandomized controlled trials. Circulation Arrhythmia and Electrophysiology. 2014;7:841-52.

32. Verma A, Jiang CY, Betts TR, et al. Approaches to catheter ablation for persistent atrial fibrillation. N Eng J Med. 2015;3 72:1812-22. 